\title{
Fuzzy Logic Based Four Step Search Algorithm for Motion Vector Estimation.
}

\author{
Suvojit Acharjee \\ Department of Electronics and Telecommunication Engineering, Jadavpur University \\ Jadavpur, Kolkata, India \\ E-mail: acharjeesuvo@gmail.com \\ Sheli Sinha Chaudhuri \\ Department of Electronics and Telecommunication Engineering, Jadavpur University \\ Jadavpur, Kolkata, India \\ E-mail: shelism@rediffmail.com
}

\begin{abstract}
Visual information is very much important for human to perceive, recognize and understand the surrounding world. As we live in the age of multimedia video sequences are very useful to us for providing information. Video involves a huge amount of data. So video compression is necessary Motion compensation has lot of computation in total video compression process. Fast motion vector estimation is a key-factor in video coding standard. Full search algorithm is the best algorithm between all the block matching algorithms to estimate the motion vector estimation with a huge computation cost. The challenge is to reduce the computational complexity of Full Search algorithm without losing too much quality at the output.

In this paper we propose to implement the fuzzy logic based Four Step Search algorithm which performs better than other block matching algorithms.

Index Terms - Four Step Search, Fuzzy logic based Four Step Search, Motion vector Estimation with Fuzzy, Fuzzy Block Matching Algorithm
\end{abstract}

\section{INTRODUCTION}

Visual information is very much important for human to perceive, recognize and understand the surrounding world. As we live in the age of multimedia video sequences are very useful to us for providing information. Video refers to the sequence of image frames. Video involves a huge amount of data. So large amount of storage is required to store a video sequence and a huge bandwidth required to transmit a video sequence. But practically this is not possible to provide a large storage or huge bandwidth for every video sequence. So video compression is a necessary step to reduce video size. Video compression refers to a process in which the amount of data used to represent the video is reduced to meet the bandwidth requirement of the network or space requirement. The ISO Moving Picture Experts Group (MPEG) video coding standards is for compressed video storage in physical media like hard disk, and International Tele-communications Union (ITU) [13] addresses the real time point to point or multipoint communication over a network. But the compression and decompression process for both the cases is almost same. Figure 1 shows the video compression process.

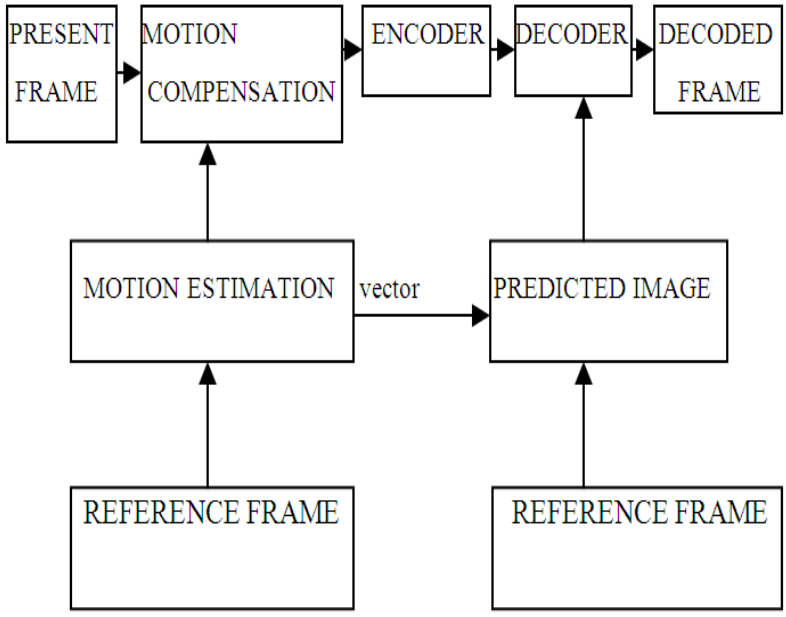

Figure 1. Video Compression Block Diagram

The motion compensation is the most computational expensive process in the whole video compression process. In motion estimation, it is assumed that change in successive frame due to translational moving of the objects. The effects of camera zoom, and object rotations are not considered. Occlusion effects are also neglected. Motion estimation examines the movement of the object in sequence to try to obtain the vectors representing the estimated motion. Encoder side estimates the motion of the current frame with respect to previous frame. A motion compensated image of the current frame is then created. Motion vector is then transmitted to decoder. Decoder reverses the whole process and creates a full frame. This way motion compensation uses the knowledge of object motion to achieve data compression. There are two techniques for motion compensated coding

- Pel-Recursive Algorithm (PRA)

- Block Matching Algorithm (BMA) 
Pel-Recursive Algorithm (PRA) [11] introduced by Netravali and Robbins, defines motion vector for every pixel which comprises the location of the pixel of the current frame in the previous frame. Block matching algorithm is the simple and straight forward technique for Motion vector Estimation. So far this technique is the most utilized technique in video compression and adopted by all the international video coding standards like ISO MPEG1, ISO MPEG2 and ITU H.264 etc. In block matching algorithm every image is partitioned into fixed size non overlapped small rectangular blocks as proposed by jain and jain [ ]. It was assumed that all the pixels within a block have same motion activity. So for every block only one motion vector is estimated. Smaller block size leads to accurate approximation but also increase the computational complexity. So a tradeoff between the accuracy and computational complexity required. So $16 \mathrm{X} 16$ can be used as standard block size. But some time for finer estimation $8 \mathrm{X} 8$ block size is used The matching of one macro block with another is based on the output of a cost function. The macro block that results in the least cost is the one that matches the closest to current block. There are various cost functions, of which the most popular and less computationally expensive is Mean Absolute Difference (MAD) [9] given by equation (1).Another cost function is Mean Squared Error (MSE) [9] given by equation (2).

$$
\begin{aligned}
& M A D=1 / N^{2} \sum_{i=0}^{N-1} \sum_{j=0}^{N-1}|\operatorname{cufra}(i, j)-\operatorname{refra}(i, j)| \\
& M S E=1 / N^{2} \sum_{i=0}^{N-1} \sum_{j=0}^{N-1}(\operatorname{cufra}(i, j)-\operatorname{refra}(i, j))^{2}
\end{aligned}
$$

$\mathrm{N}=$ Size of the macro block.

$\operatorname{cufra}(i, j)=$ Intensity value for the current frame.

$\operatorname{refra}(i, j)=$ Intensity value for the reference frame

Signal to Noise ratio (SNR) is widely used in quality measurement. SNR can be calculated using equation (3).

$$
S N R=10 \log \left(\frac{\sum_{k=0}^{V-1} \sum_{l=0}^{H-1} \text { limgout }(k, l)^{2}}{V H . M S E_{\text {ms }}}\right)
$$

In image quality measurement another closely related term is Peak Signal to Noise Ratio (PSNR) which is a modification of SNR. PSNR characterizes the motion compensated image that is created by using motion vectors and macro blocks from the reference image. PSNR can be calculated using equation (4).

$$
P S N R=10 \log \left[\frac{(\text { PtoP })^{2}}{M S E}\right]
$$

$\mathrm{PtoP}=$ peak to peak signal $\mathrm{MSE}=$ Mean square error

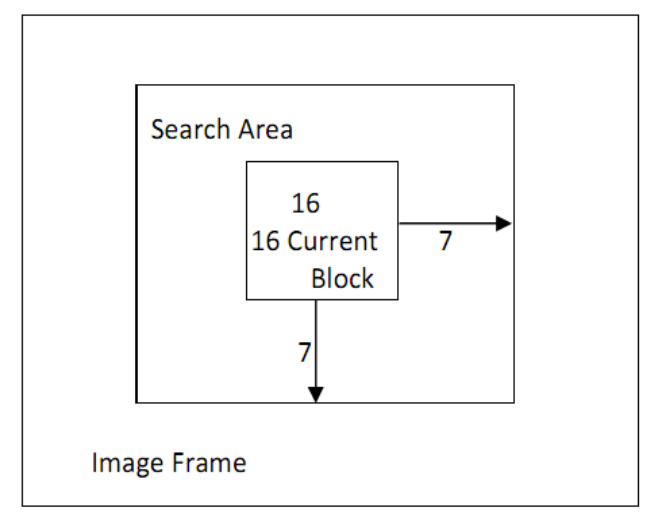

Figure 2. Block Matching of a 16x16 macro Block within a search area of 7 pixels.

Block matching algorithm for motion estimation is accepted in all the video coding standards proposed till date. It is easy to implement in hardware and real time motion estimation and prediction is possible. Exhaustive search (Full Search) is the most time and computationally expensive algorithm. Later the other algorithms implemented to reduce the computation cost are Three Step Search (TSS), Four Step Search (FSS), New Three Step Search (NTSS), Diamond Search (DS), and Adaptive Rood Pattern Search (ARPS) etc. In this paper we propose to implement Fuzzy logic based Four Step Search Algorithm.

Section I is a brief introduction about the motion vector estimation and video compression. Section II is a short review on the different research works about motion compensation. Section III describes the Four Step Search algorithm. Section IV presents our proposed algorithm. In Section V the performance of our proposed algorithm is compared with different block matching algorithms.

\section{Literature REVIEW}

Pel-recursive algorithm, is the first algorithm developed by Robbins and Netravali, defines motion vector for every pixel with the help of steepest decent method. Bargman [14] proposed to use Newton Raphson method instead of using steepest descent method. Walker and Rao [10] introduced a variable step size for steepest descent method.

For block matching algorithms, Full search or Exhaustive search provides best result. Later different algorithms were developed to reduce the computation cost of Full search algorithms. Earlier square search pattern of different length was adopted. Algorithms like Three Step Search [2], New Three Step Search [3], Four Step Search [4], Block Based Gradient Descent Search [5], Simple and Efficient Search [12] etc have adopted the square search pattern. The square search pattern can be classified in ' + ' and ' $x$ ' pattern. Two dimensional logarithmic search [1] has adopted the ' + ' search pattern where cross search algorithm [17] has adopted ' $x$ ' search pattern. 
Then diamond type search pattern minimizes the process time. Different algorithms adopted the diamond search pattern as unrestricted center biased diamond search algorithm [8], New Diamond Search Algorithm [6] etc. Adaptive Rood Pattern Search [16] uses the motion vector of the macro block to its immediate left to predict the motion vector of the current macro block. This algorithm uses the diamond type search pattern. But ARPS performs better than other algorithms. Recent studies show that other patterns like hexagon [7], octagon [15] provides better result than diamond pattern. Other approaches to reduce the computation cost of Full search are Fast full search algorithm [19] and Successive elimination algorithm [18] etc.

\section{Four Step Search Algorithm}

In the year 1996 four step search algorithm proposed based on the center biased motion vector distribution characteristics by LaiMan Po and Wing-Chung Ma. The Four Step Search (FSS) algorithm can be summarized as follows-

[1] Set search parameter 7. As shown in figure 3a, pick nine points from $5 \times 5$ window located at previous frame to start search for the best match.

[2] Measure the block distortion with any of the cost function between the block in the current frame and the block to be search for best match at the previous frame.

[3] Find the block with minimum distortion.

[4] If the minimum distortion is at the center. Then go to step 8 .

[5] Else the search continues. Search window remains 5X5. The search pattern depends on the position of previous minimum distortion point.

Step A, If the minimum distortion point is at the corner then five additional points need to be search as shown in figure $3 b$.

Step B, Else the minimum distortion point is at the middle of the vertical or horizontal axis then three additional points need to be search as shown in figure $3 \mathrm{c}$.

[6] If the minimum distortion point is at center then go to step 8 else go to next step.

[7] The search process is same like step 5 and step 6 . The process continues to step 8 .
[8] Search window reduced to $3 \mathrm{X} 3$ as shown in figure $3 \mathrm{~d}$. Additional eight points need to be search. The minimum distortion point from these nine points is the best match.

If the minimum distortion point is at the center then The intermediate step of the algorithm may be skipped and we can move to final step. So, computational requirement can be reduced. Total no of checking points varies from 17 to 27.

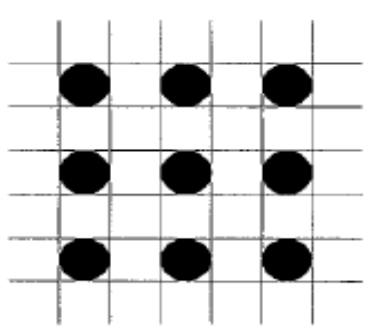

(a)

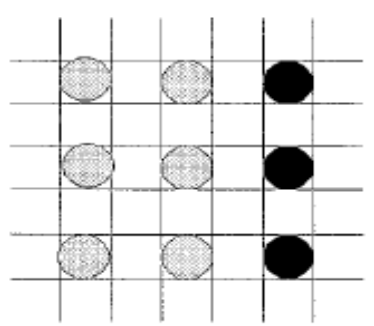

(c)

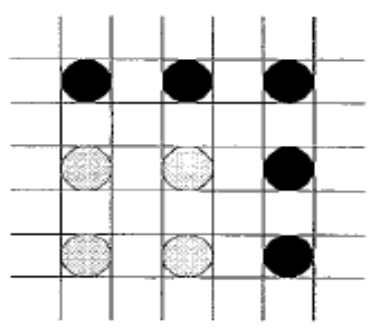

(b)

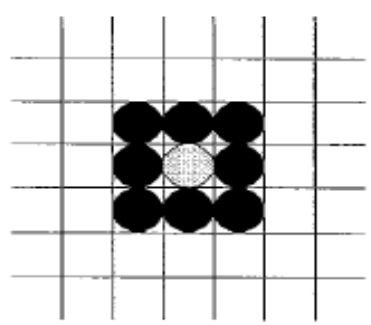

(d)
Figure 3. Search Patterns for FSS (a) Step one and two (b) Step Five A (c) Step Five B (d) Step Eight

\section{Proposed Algorithm}

Four step search algorithm searches all the four side of a macro block. But some time search at all the four side is not necessary. The change from dark intensity to light intensity is called edge region in an image. A macro block in one side of an edge need not be search for its best match at the other side of the edge. If the macro block is at the lighter side of the edge then search at the darker side for best match is not necessary. According to proposed algorithm a fuzzy membership value introduced according to intensity for every block. From the intensity values of the pixels within a macro block a value is calculated which defines whether the macro block is in darker or lighter region. Search can only continue if the fuzzy membership value of the macro block of the reference frame is in the allowable range of macro block of the current frame. The search pattern and other step is similar like four step search.

[1] Calculate fuzzy membership value for every block in the previous frame

[2] Calculate fuzzy membership value for every block in the current block. 
[3] Set the search location at center of search window and set search window parameter 7 .

[4] Pick nine points from the $5 \times 5$ window located at previous frame to start search for the best match.

[5] Whether the fuzzy membership value of the block of reference frame going to be search is in the allowable range with the fuzzy membership value of the present frame.

[6] If yes then calculate the block measure distortion for that macro block else skip the calculation.

[7] Find the minimum block distortion between those macro blocks whose block distortion has been calculated.

[8] If the minimum distortion is at the center then go to step 14.

[9] Else search continues. Search window remains $5 \mathrm{X} 5$. The search pattern depends on previous minimum distortion point.

Step A, If the point is at the corner then pick five additional points as shown in figure $3 b$.

Step B, If the minimum distortion point is at the middle of the vertical or horizontal axis then pick three additional points as shown in figure $3 \mathrm{c}$.

[10] The search process will continue for the additional points only if the condition stated at step five is yes. Else skip the search for that macro block.

[11]Find the minimum block distortion between those macro blocks whose block distortion has been calculated.

[12] If the minimum distortion is at the center then go to step 14. Else go to next step.

[13] The search process is same like step nine, ten and eleven. Process continues to step 14.

[14] Search window reduced to $3 X 3$. Pick additional eight points.
[15] Search process only continues if the condition stated in step five valid for the points else we skip the search.

[16] From the calculated block distortion measure, the block with minimum distortion is the best match.

Calculated motion vector saved for transmission. Same as four step search if the minimum distortion point is at the center then the intermediate steps can be skipped. In this whole process by adding the additional fuzzy membership value those calculations can be skipped which are not necessary. The fuzzy membership value can be anything that defines whether the block is lighter or darker. The mean of the intensity values of macro blocks can be used as fuzzy membership value. The mean value is normalized between 0 to 1 .

Mean $=1 /\left(255 * N^{2}\right) \sum_{i=0}^{N-1} \sum_{j=0}^{N-1} \operatorname{frame}(i, j)$

$\mathrm{N}=$ Size of the Macro Block

frame $(i, j)=$ intensity value at $\mathrm{i}, \mathrm{j}$.

As 255 is the maximum intensity for an image. So this value is needed to normalize the value of mean between 0 to 1 .

This algorithm has a worst computational requirement is 27 . But the unnecessary computations can be skipped using the fuzzy membership value. So minimum required computation can be reduced than four step search algorithm. This will increase the speed of the process. The speed of the process and the quality of the reconstructed image can be controlled by controlling the allowable range. For higher speed a narrow range is required and for good quality wider range need to set.

\section{Simulation}

Proposed algorithm simulated using first six frames of the foreman sequence. The performance of the proposed algorithm is then compared with the performance of other block matching algorithm as Three Step Search (TSS), New Three Step Search (NTSS), Four Step Search (FSS) etc. In the simulation procedure MSE is used as cost function to determine the block distortion. Search window parameter is 7 . In the video compression after few frames one frame is need to be sent in original. But here we use only siz frames so first frame is sent in original. Figure $4,5,6$, shows the $4^{\text {th }}$ no of frame in original, reconstructed after FSS procedure and reconstructed after proposed algorithm. 


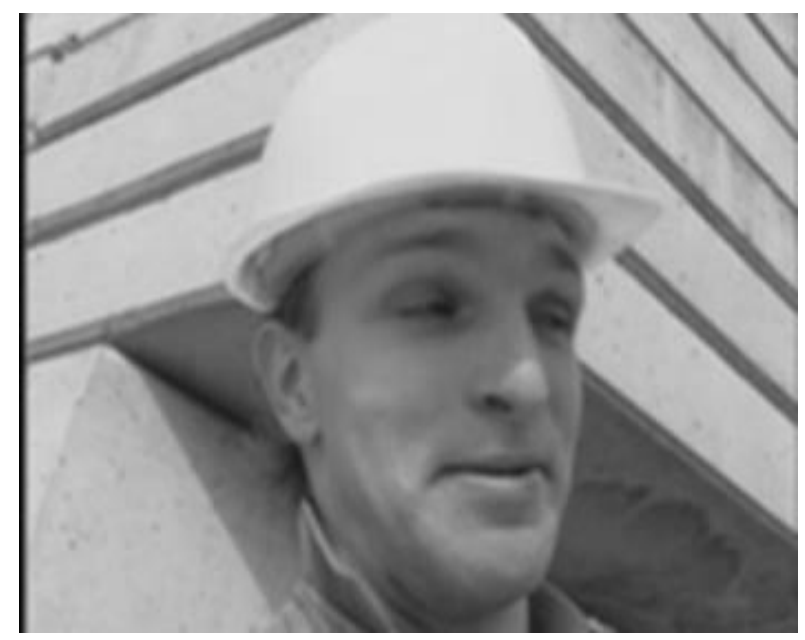

Figure 4. Original foreman sequence $4^{\text {th }}$ frame.

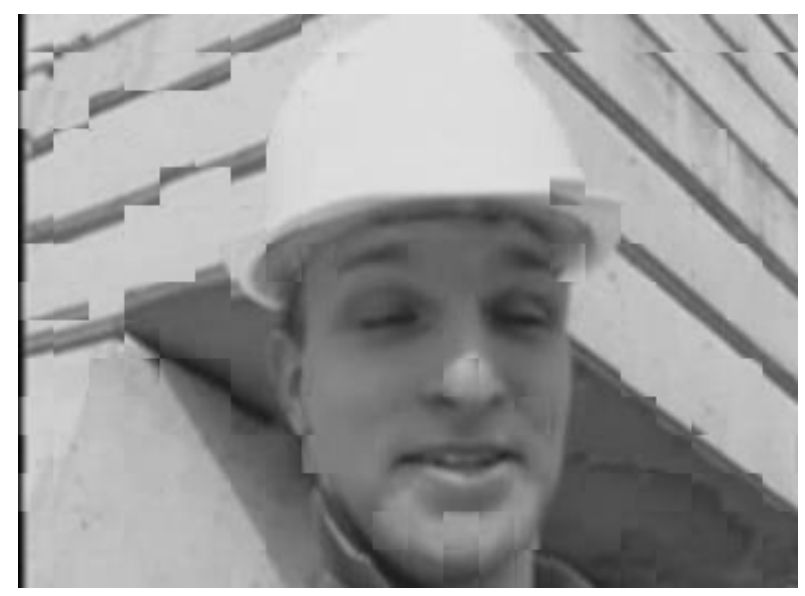

Figure 5. After FSS procedure reconstructed foreman Sequence $4^{\text {th }}$ frame.

In Figure 7, 8, 9 we compare the performance of the proposed algorithm with other block matching algorithms. It shows with almost same picture quality

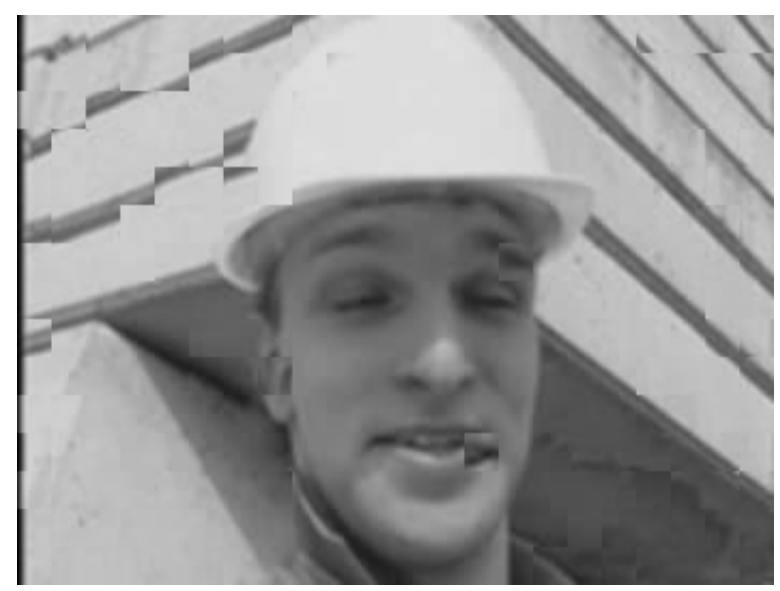

Figure 6. After Fuzzy logic based FSS procedure reconstructed foreman sequence $4^{\text {th }}$ frame.

proposed algorithm performs better than other block matching algorithm in terms of Time and computation.

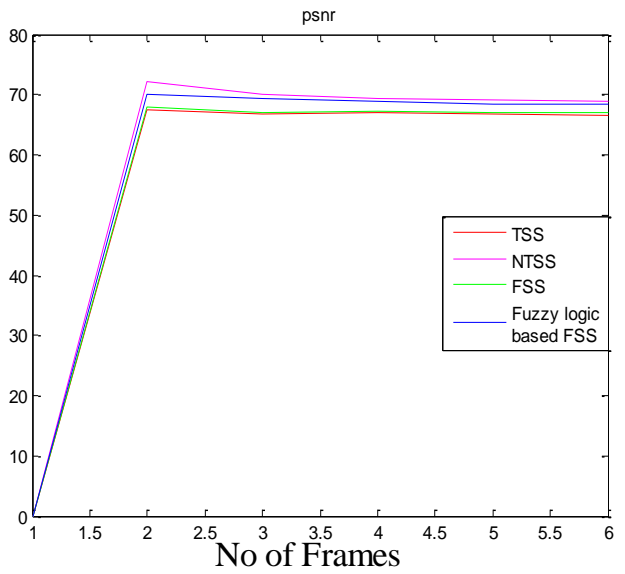

Figure 7. PSNR performance by different algorithms

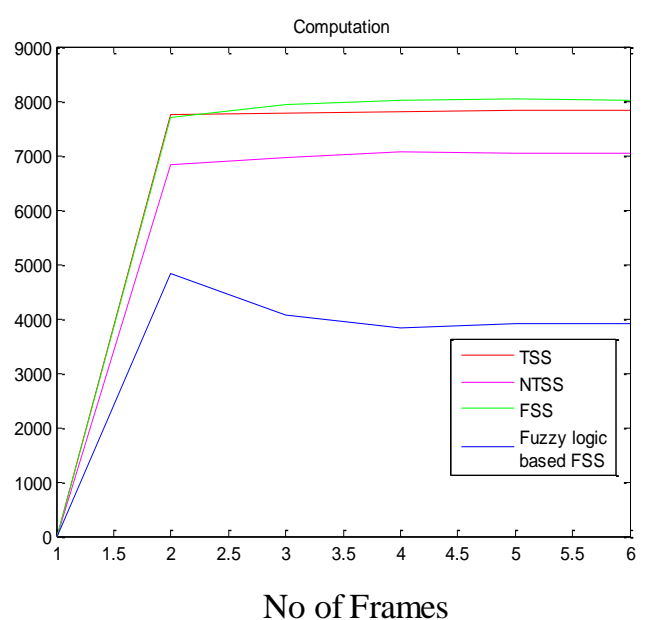

Figure 8. Total Computation required by different algorithms

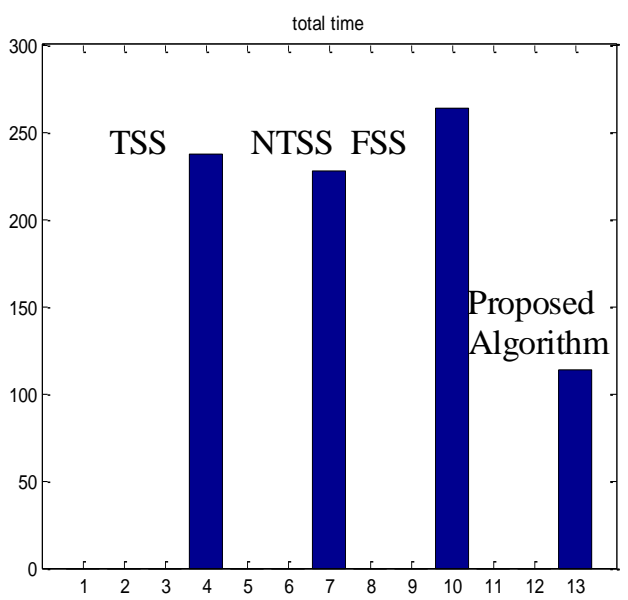

Figure 9. Total time in Seconds required for different algorithms 


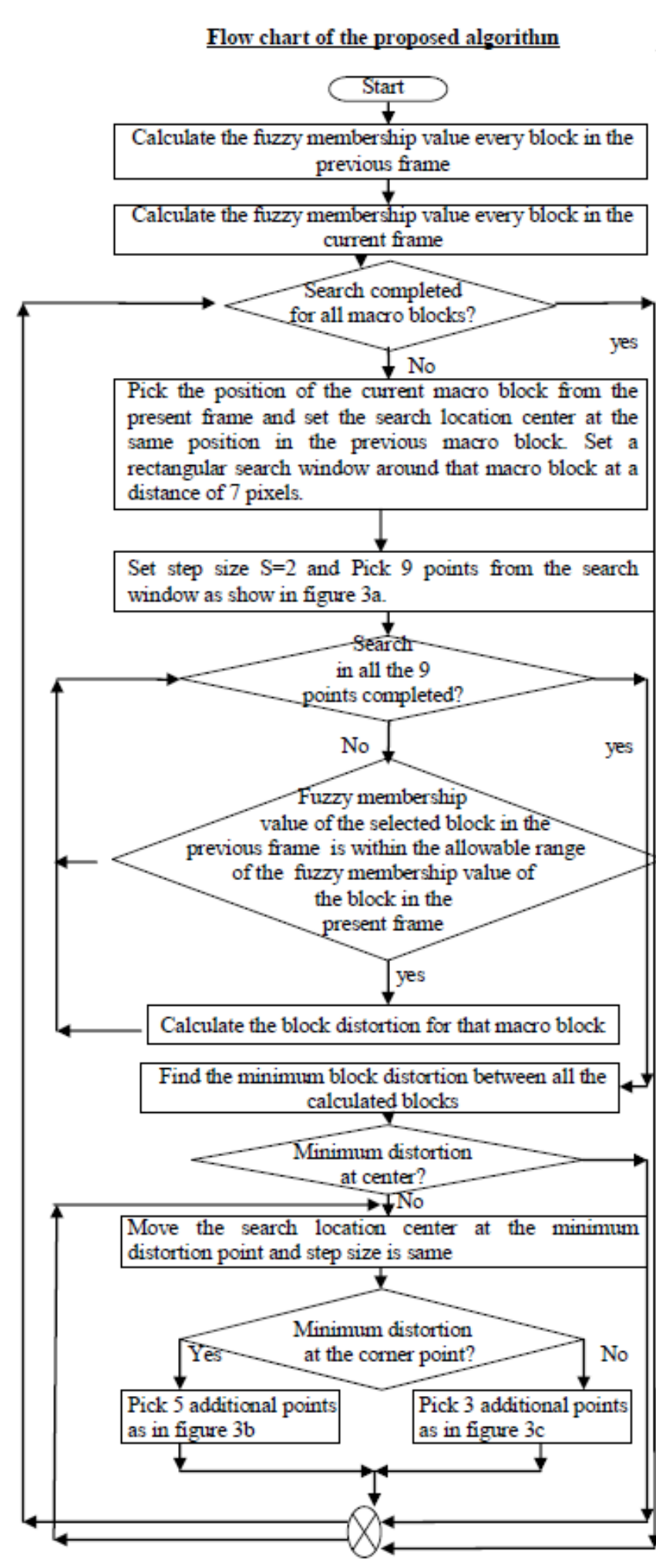

\section{Conchusion}

Fuzzy logic based four step search algorithm reduces the computation. As the computation reduced the whole process becomes faster. In this algorithm the speed of the process and the quality of the reconstructed image

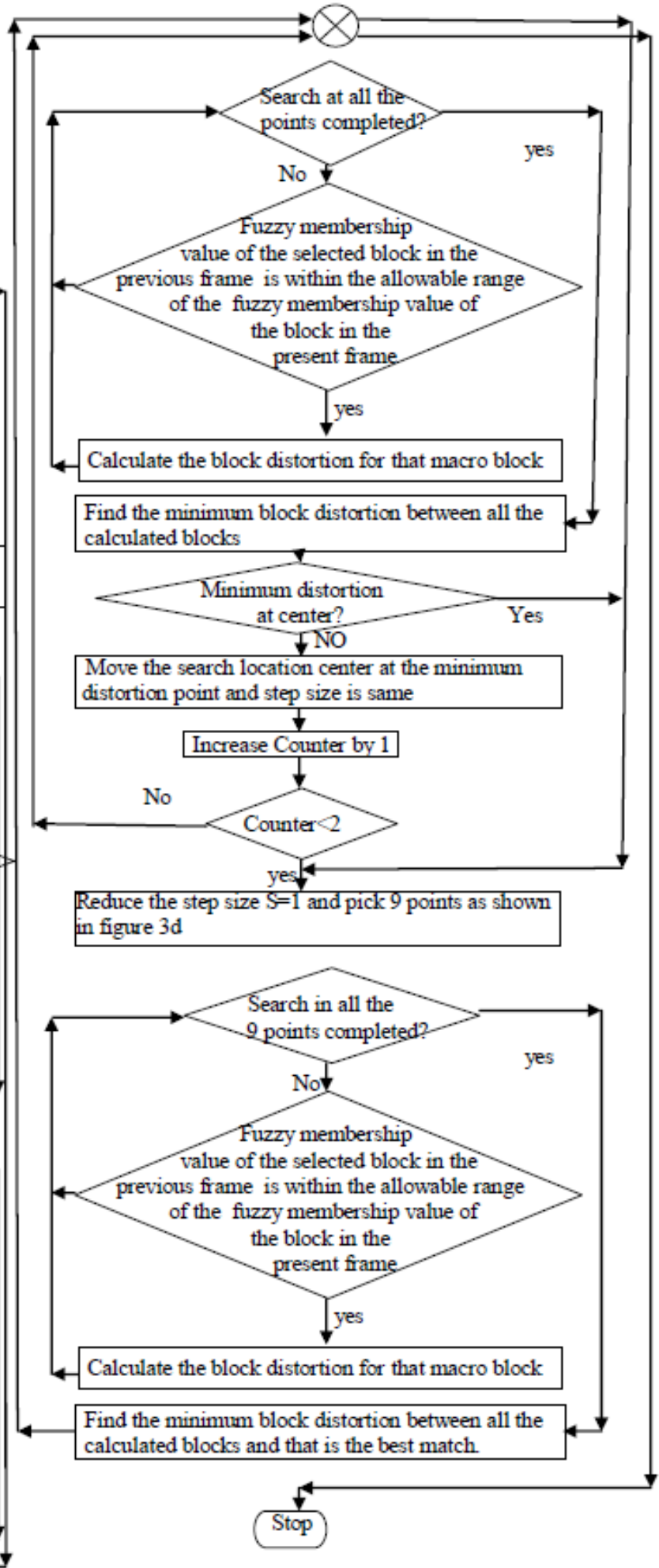

can be controlled. This concept can be applicable for other block matching algorithms.

\section{REFERENCES}

[1] J. Jain and A. Jain, "Displacement measurement and its application in interframe image coding," IEEE 
Trans. Commun., vol. COMM-29, pp.1799-1808, Dec. 1981.

[2] T. Koga, K. Iinuma, A. Hirano, Y. Iijima, andT. Ishiguro, "Motion compensated interframe coding for video conferencing," in Proc. National Telecommunication. Conf., New Orleans, LA, Nov.29-Dec. 3 1981, pp. G5.3.1-.3.5.

[3] R. Li, B. Zeng, and M. L. Liou, "A new three step search algorithm for block motion estimation," IEEE Trans. Circuits Syst. Video Technol., vol. 4, pp. 438-442, Aug. 1994.

[4] L. M. Po and W. C. Ma, "A novel four-step search algorithm for fast block motion estimation," IEEE Trans. Circuits Syst. Video Technol., vol. 6, pp. 313-317, June 1996.

[5] L. K. Liu and E. Feig, "A block-based gradient descent search algorithm for block motion estimation in video coding," IEEE Trans. Circuits System Video Technol., vol. 6, pp. 419-423, Aug. 1996

[6] Shan Zhu, and Kai-Kuang Ma, “ A New Diamond Search Algorithm for Fast Block-Matching Motion Estimation", IEEE Trans. Image Processing, vol 9, no. 2, pp. 287-290, February 2000.

[7] Ce Zhu, Xiao Lin, and Lap-Lui Chau,"Hexagon based Search Pattern for Fast Block Motion Estimation ", IEEE Trans. On circuits and systems for video technology, Vol.12, No.5,May 2002

[8] J.Y. Tham, S. Ranganath, M. Ranganath, A.A.Kassim, "A novel unrestricted centerbiased diamond search algorithm for block motion estimation", IEEE Trans. Circuits Systems VideoTechnol. 8 (August 1998) 369-377.

[9] Y.Q.Shi and H Sun, "Image and Video Compression for Multimedia Engineering “"

[10] Walker D.R., Rao.K.R. "Improved Pel-Recursive Motion Compensation", IEEE trans on Communications, vol com 32,no. 10, Oct. 1984, 1128-1134.

[11]A.N.Netravali and J.D.Robbins, "Motion compensated television coding-part1", Bell syst. Tech.J., Vol.58,pp 631-670,Mar.1979

[12] Jianhua Lu amd Ming L.Liou “ A Simple and Efficient search algorithm for Block Matching Motion Estimation", IEEE trans circuits And Systems for Video Technology, Vol 7,no,2,pp. 429433 april 1997

[13]A Barjatya, "Block Matching Algorithms For Motion Estimation," DIP 6620 Spring 2004 Final Project Paper

[14]H.C.Bergmann, "Displacement estimation based on the correlation of image", IEEE proceedings of International conference on Image processing,York,England,july 1982,pp. 215-219.

[15]L.P.Chau and C.Zhu, "A fast octagon-based search algorithm for motion estimation", Elsevier Science Signal processing 83,2003,pp. 671-675.

[16] Yao Nie and Kai Kuang Ma, "Adaptive Rood Pattern Search for Fast Block Matching Motion Estimation", IEEE transaction on Image Processing, Vol 11, no. 12,1442-1448,December 2002.
[17]M. Ghanbari, “ The Cross Search Algorithm for motion Estimation", IEEE Transaction on Communication vol. 38, pp. 950-953, july 1990.

[18] W. Li and E. Salari, "Successive elimination Algorithm for motion Estimation", IEEE transaction on Image Processing ,Vol 4,no 1,pp. 105107,January 1995.

[19]Y.C. Lin and S.C. Tai, "Fast Full Search Block matching algorithm for motion compensated video compression", IEEE transactions on Communications, Vol 45, no. 5 pp. 527-531, May 1997

Suvojit Acharjee was born in Murshidabad, West Bengal, Indi on March $16^{\text {th }}, 1988$. Suvojit is now a Mtech student in Jadavpur University. Suvojit is awarded the degree of Bachelor of Technology in recognition of his successful completetion of of the 4 year programmed Electronics and Communication course from Future Institute of Engineering and Management, Kolkata under the university West bengal University of Technology, Kolkata, India on June , 2009.

His current research interests include Robotics, Soft Computing, Image Processing, Video Compression and algorithms.

Dr. Sheli Sinha Chaudhri is an associate professor of Jadavpur University. Her research interests include soft computing, Image processing, Multimedia. 